\title{
Elemental bio-imaging of melanoma in lymph node biopsies
}

Dominic Hare ${ }^{1}$, Fiona Burger ${ }^{1}$, Christine Austin ${ }^{1}$, Fred Fryer $^{2}$, Rudolf Grimm ${ }^{3}$, Brian Reedy ${ }^{1}$, Richard A Scolyer ${ }^{4}$, John F Thompson ${ }^{4} \&$ Philip Doble ${ }^{1 *}$

${ }^{1}$ Elemental Bio-imaging Facility, University of Technology, Sydney, Broadway, New South Wales, Australia

${ }^{2}$ Agilent Technologies Australia, North Ryde, New South Wales, Australia

${ }^{3}$ Agilent Technologies Inc., Santa Clara, California, USA

${ }^{4}$ Sydney Melanoma Unit and Melanoma Institute Australia, Royal Prince Alfred and Mater Hospitals, Sydney, New South Wales, Australia

* Corresponding author, Department of Chemistry and Forensic Science, University of Technology, Sydney, 1 Broadway, Broadway, NSW, 2007, Australia, Ph. +61 29514 1792, Fax. +61 29514 1460, Email.philip.doble@uts.edu.au

\begin{abstract}
The spatial distribution of trace elements in human lymph nodes partially infiltrated by melanoma cells was determined by elemental bio-imaging. Imaging of ${ }^{31} \mathrm{P}$ within the nodal capsule and normal lymph node tissue showed a clear demarcation of the tumour boundary, with a significant decrease in relative ${ }^{31} \mathrm{P}$ concentration within the tumour. The location of the tumour boundary was confirmed by haematoxylin and eosin staining of serial sections and observation by light microscopy. Further enhancement of the tumour boundary was achieved by imaging the ${ }^{31} \mathrm{P} /{ }^{34} \mathrm{~S}$ ratio. ${ }^{31} \mathrm{P} /{ }^{66} \mathrm{Zn}$ ratio images showed a decreasing ratio beyond the tumour boundary that extended into peritumour normal lymph node tissue.
\end{abstract}

\section{Introduction}

Melanoma is a malignant tumour that arises from melanocytes that are normally found in the basal cell layer of the epidermis of the skin. The WHO estimated the global mortality from melanoma in 2000 at over 65,000 ${ }^{1}$. 9,722 new cases of melanoma were diagnosed in Australia in 2004, with 1,200 reported fatalities $^{2}$ giving Australia the highest incidence of melanoma in the world. It has been suggested that global incidence rates of melanoma will double every 10-20 years for the foreseeable future ${ }^{3}$.

Mortality from most solid malignancies usually occurs as a consequence of tumour metastasising from its site of origin to distant locations. Lymph nodes receive lymphatic fluid directly from the primary tumour and are the most common first sites to harbour metastases. Metastatic melanoma is present in lymph nodes in approximately $20 \%$ of melanoma patients with primary tumours $\geq 1 \mathrm{~mm}$ in thickness ${ }^{4}$. 
Identification of the presence of melanoma metastases in clinically negative regional lymph nodes is usually achieved by surgical removal of the lymph node most likely to harbour metastases (i.e. the sentinel lymph node) and laborious histopathological examination of tissue sections stained both with hematoxylin and eosin (H\&E) and immunohistochemically for various melanoma-associated antigens. The tumour-harbouring status of the sentinel lymph node is the most important prognostic factor in patient's presenting with early stage melanoma ${ }^{5}$.

An alternative method for detection of melanoma in lymph nodes is chemical imaging. Fourier transform infrared (FTIR) spectral imaging has been used to investigate cutaneous melanomas ${ }^{6,7}$. Recently, $K$-means clustering was used to identify infrared spectral similarities in skin basal cell carcinomas (BCCs), highlighting the utility of imaging technology and chemometrics for the diagnosis of cutaneous malignancies ${ }^{8}$.

Although trace elements have long been associated with malignancy, their role and prevalence in melanoma is unknown. Phosphorus/sulphur ratios have been shown to be higher in malignant human hepatocarcinoma cell lines when compared to normal human dermal fibroblast and transformed embryo kidney cell lines ${ }^{9}$. Sulphur-based, cysteine-rich, zinc-binding metallothioneins (MTs) have shown both increased expression and down regulation in numerous types of types of cancers ${ }^{10}$. Increased copper concentrations and lower iron levels were reported following the analysis of toenail clippings from 58 newly diagnosed cutaneous melanoma patients who did not show evidence of metastasis compared with 58 control samples ${ }^{11}$. In another study, serum trace element levels in healthy controls and patients with varying stages of melanoma showed an increase in zinc levels. However, no statistical difference in serum copper levels was noted in the melanoma patients ${ }^{12}$.

Imaging mass spectrometry ${ }^{13}$ employs matrix-assisted laser desorption/ionisation mass spectrometry (MALDI-MS) for the imaging of peptides in tissues by monitoring specific mass-to-charge $(\mathrm{m} / \mathrm{z}$ ) ratios. This technique has been applied to glioma biopsies and numerous other diseases ${ }^{14}$. In a similar manner, elemental bioimaging of tissue sections is possible by employing laser ablation-inductively coupled plasma-mass spectrometry (LA-ICP-MS) ${ }^{15}$. LA-ICP-MS is capable of determining most of the elements with a 7 order magnitude linear dynamic range, with detection limits as low as parts per trillion. The system utilises a focused laser beam (10 to 200um diameter) directed onto the surface of the tissue. The sample is ablated by an energy transfer from the laser, resulting in a plume of particles swept to the ICP by a carrier gas. The spatial distribution of elements across a tissue sample can be determined by collecting mass spectra as the laser traverses the sample in two dimensions. Maps can then be constructed of both metals and non-metals essential to physiology. Recently, elemental bioimaging has been applied to human glioblastomas ${ }^{16}$, labelled $\beta$-amyloid-bound antibodies in Alzheimer's disease $^{17}$, implanted tumour cells ${ }^{18}$ and $\mathrm{Cu}, \mathrm{Zn}$ and Pt in cis-platin dosed mice ${ }^{19}$. 
The current study demonstrated the potential of elemental bioimaging for detection of metastatic melanoma in lymph nodes in human tissues. Construction of maps presented as single elements or ratios of elements, provided clear images of the melanoma and adjacent non-tumorous tissues.

\section{Experimental}

\section{Instrumentation}

Experiments were conducted using a New Wave UP-213 laser ablation unit (Kenelec Technologies, Mitcham, Victoria, Australia) hyphenated to a 7500ce ICP-MS (Agilent Technologies, Forrest Hill, Victoria, Australia). A 'cs' lens system was fitted to the 7500ce for enhanced sensitivity. The frequency-quintupled Nd:YAG laser produced a laser wavelength of $213 \mathrm{~nm}$. The LA-ICP-MS system was tuned for sensitivity using NIST 612 glass.

Apart from high sensitivity, the instrument was tuned to deliver low oxides. This was done by ablating NIST 612 glass and measuring masses 232 and 242 (Th and ThO respectively). Using an RF power setting of $1200 \mathrm{~W}$ and sampling depth of $6 \mathrm{~mm}$, ThO/Th ratios were consistently lower than $0.3 \%$. The low oxides are attributed, in part, to the laser generated aerosol being essentially dry. In standard sample introduction systems, relatively large amounts of solvent (typically water) are passed into the plasma, contributing large amounts of oxygen and lowering the effective plasma temperature. Low ThO\Th ratios contributed to generally low matrix derived matrix interferences. Low sample load from the ablated tissue minimised the influence of oxide-based interferences from water in the sample matrix. Measurement of ${ }^{32} \mathrm{~S}^{+} /{ }^{34} \mathrm{~S}^{+}$and ${ }^{56} \mathrm{Fe}^{+} /{ }^{57} \mathrm{Fe}^{+}$ratio in tissue sections confirmed the natural isotopic abundance ratios.

Table 1: LA-ICP-MS operation parameters.

\begin{tabular}{|l|l|l|l|}
\hline \multicolumn{2}{|l|}{ Agilent 7500ce ICP-MS } & \multicolumn{2}{l|}{ New Wave UP213 Laser Ablation } \\
\hline Rf Power & $1200 \mathrm{~W}$ & Wavelength & $213 \mathrm{~nm}$ \\
\hline Cooling gas flow rate $15 \mathrm{~L} \mathrm{~min}^{-1}$ & Repetition frequency & $20 \mathrm{~Hz}$ \\
\hline Carrier gas flow rate & $1.1 \mathrm{~L} \mathrm{~min}^{-1}$ & Laser energy density & $0.3 \mathrm{~J} \mathrm{~cm}^{-2}$ \\
\hline Sample depth & $6.0 \mathrm{~mm}$ & Spot size & $40 \mu \mathrm{m}$ \\
\hline QP Bias & $-5 \mathrm{~V}$ & Scan rate & $40 \mu \mathrm{m} \mathrm{s}^{-1}$ \\
\hline OctP Bias & $-8 \mathrm{~V}$ & Line spacing & $40 \mu \mathrm{m}$ \\
\hline Scan mode & Peak hopping & Carrier gas & $\mathrm{Ar}$ \\
\hline Dwell time & $0.1 \mathrm{~s}$ per m/z & & \\
\hline Measured m/z & $13,31,32,34,56,57,63,66$ & & \\
\hline Extracts 1,2 & $6.8,-126 \mathrm{~V}$ & & \\
\hline
\end{tabular}

\section{Image construction}

A laser spot size of $40 \mu \mathrm{m}$ and $40 \mu \mathrm{m} \mathrm{s}^{-1}$ scan was employed to construct the images. Under these conditions the recorded counts were at least 10 times above baseline. Image quality was dependant on 
both the integration time of the MS and the number of elements measured. An integration time of $0.1 \mathrm{~s}$ per mass was adequate for the 8 chosen isotopes, ${ }^{31} \mathrm{P},{ }^{66} \mathrm{Zn},{ }^{13} \mathrm{C},{ }^{32,34} \mathrm{~S},{ }^{56,57} \mathrm{Fe}$ and ${ }^{63} \mathrm{Cu}$. The $0.1 \mathrm{~s}$ integration time and $40 \mu \mathrm{m} \mathrm{s}^{-1}$ scan speed resulted in an approximately $4 \mu \mathrm{m}$ shift from the original location of ablation. Scan speed, spot size and integration time were selected in order to generate a true image resolution of approximately $40 \mu \mathrm{m}$ per pixel. Images were acquired over an approximately 18hour period. The images were constructed as colour maps of relative concentration after normalisation to ${ }^{13} \mathrm{C}$. That is the signal from the isotope of interest was divided by that of ${ }^{13} \mathrm{C}$ to compensate for differences in tissue thickness and density, as reported previously ${ }^{15}$. Relative concentrations were suitable as the concentration differences of the isotopes were easily visualised across each lymph node.

LA-ICP-MS data was exported in comma separated variable (CSV) file formats with a separate file created for each line of ablation in the $x$-axis. A Visual Basic macro was used to both normalise each data point to ${ }^{13} \mathrm{C}$ and combine all resultant data into a single ASCII file. This file was then imported into the ITT Visual ENVI 4.2 (Research Systems Inc., Boulder, CO, USA) hyperspectral imaging software package, where final images were produced.

\section{Lymph node samples}

The study was approved by the institutional review boards of the Royal Prince Alfred Hospital and the University of Technology, Sydney. Patients with metastatic melanoma in excised lymph nodes were identified from the Sydney Melanoma Unit database. The histological slides and the formalin-fixed, paraffin-embedded tissue blocks of the lymph node specimens were retrieved from the archives of the Department of Anatomical Pathology at the Royal Prince Alfred Hospital, Sydney, Australia. Following review of the original sections, a representative tissue block was selected and section at 5 $\mu \mathrm{m}$, then mounted onto slides. The sections were dewaxed with high purity xylene, ethanol (Sigma Aldrich, Castle Hill, NSW, Australia) and $18.2 \mathrm{~m} \Omega$ water (Sartorius, East Oakliegh, Victoria, Australia). Serial sections were stained with $H \& E$ and immunohistochemically for melanoma associated antigens S-100 protein, HMB-45 and Melan A. Histological examination by a histopathologist (RAS) confirmed the presence of metastatic melanoma. Examination of the unstained section by the laser's CCD camera revealed no notable difference in surface structure across the sections.

\section{Results and discussion}




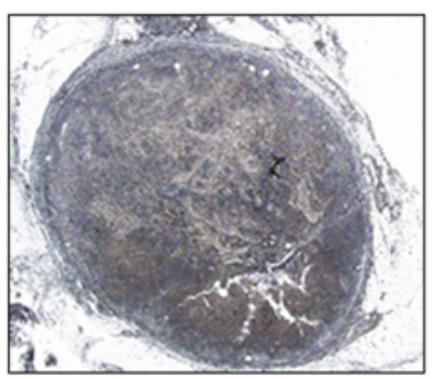

(a) Micrograph

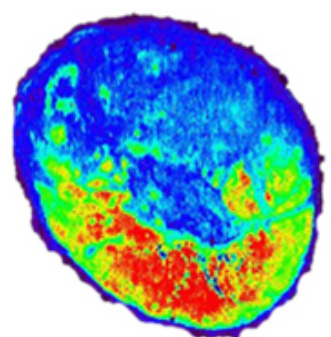

(d) ${ }^{31} \mathrm{P}^{+}$

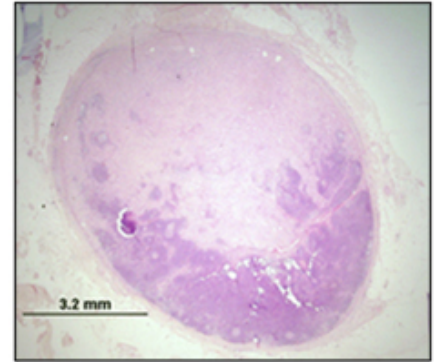

(b) H \& E Stain

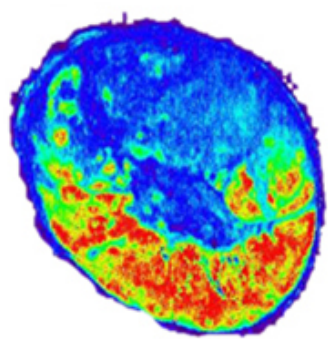

(e) ${ }^{31} \mathrm{P}^{+} / 34 \mathrm{~S}^{+}$

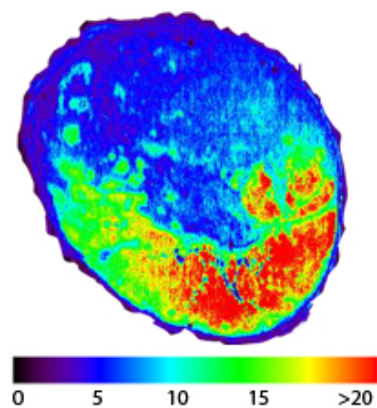

(c) ${ }^{31 \mathrm{P}}+\left(\right.$ no $\left.{ }^{13} \mathrm{C}\right)$

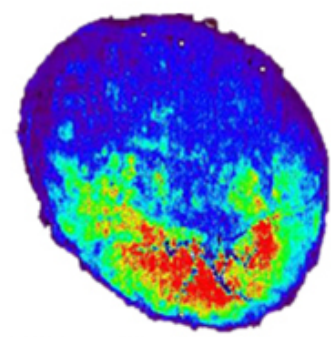

(f) ${ }^{31} \mathrm{P}^{+} / 66 \mathrm{Zn}^{+}$

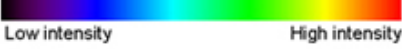

Figure 1: Sentinel lymph node partly replaced by metastatic melanoma (upper two thirds of the ovoidshaped lymph node). (a) Photograph of slice before elemental imaging, (b) $\mathrm{H}+\mathrm{E}$ stain, (c) ${ }^{31} \mathrm{P}$ image, (d) ${ }^{31} \mathrm{P} /{ }^{34} \mathrm{~S}$ and (e) ${ }^{31} \mathrm{P} /{ }^{66} \mathrm{Zn}$

Figure 1(a) is a light micrograph of a lymph node containing metastatic melanoma. The image is not uniform in colour, with slightly darker pigmentation in the lower half. The presence of metastatic melanoma could not be detected by visual inspection. The tissue also appeared to be of uniform density when viewed with a light microscope. Figure 1(b) shows an H\&E stain of a section cut immediately adjacent to the section shown in Figure 1(a) and shows metastatic melanoma involving about two thirds of the lymph node. H\&E stained sections have been reported as suitable for the detection of melanoma micrometastases ${ }^{20}$. The pale areas (predominantly in the upper two thirds of the ovoid lymph node) represent metastatic melanoma.

Figure 1 (c) is a ${ }^{31} \mathrm{P}$ image prior to ${ }^{13} \mathrm{C}$ normalisation. There was variation in the concentration of phosphorus that corresponded to the presence of the tumour as determined by the $\mathrm{H} \& \mathrm{E}$ stain. Figure $1(\mathrm{~d})$ is the same image normalised to ${ }^{13} \mathrm{C}$. There was significant improvement in contrast in the normalised image, particularly on the left side of the section. This normalisation process also compensated for varying thickness in the tissue slice.

There were no significant variations in concentrations of ${ }^{32,34} \mathrm{~S},{ }^{56,57} \mathrm{Fe},{ }^{63} \mathrm{Cu}$ and ${ }^{66} \mathrm{Zn}$ when imaged as separate isotopes (data not shown). Figure 1 (e) clearly shows an increased ${ }^{31} \mathrm{P}^{+} /{ }^{34} \mathrm{~S}^{+}$ratio in the peritumoural lymphoid tissue when compared with the metastatic tumour. Furthermore, the ${ }^{31} \mathrm{P}^{+} /{ }^{34} \mathrm{~S}^{+}$ ratio improved contrast between the melanoma and lymphoid cells, when compared with ${ }^{31} \mathrm{P}^{+}$images alone. Construction of ${ }^{31} \mathrm{P}^{+} /{ }^{66} \mathrm{Zn}^{+}$ratios (Figure 1 (f)) shows a slightly different perspective on the tumour boundary. The blue regions (relatively lower values) penetrated further into the peritumoural, 
apparently uninvolved, lymphoid tissue (determined from the H \& E stain) than in the images of the ${ }^{31} \mathrm{P}$ and the ${ }^{31} \mathrm{P}^{+} \beta{ }^{34} \mathrm{~S}^{+}$ratio. These results suggest changes in biochemistry of the peritumoural lymphoid tissue that likely represents a response to the tumour.

Figure 2 shows a 20-x magnification of the tumour boundary overlayed with the corresponding ${ }^{31} \mathrm{P}$ image. Clear evidence of metastatic melanoma was observed on the H\&E stained section (the pale area in the upper right portion of the lymph node in Fig. 1.b) confirmed by immunohistochemistry. The boundary between healthy and cancerous cells correlated well with the changing intensities of ${ }^{31} \mathrm{P}$, ${ }^{31} \mathrm{P} /{ }^{34} \mathrm{~S}$ and ${ }^{31} \mathrm{P} /{ }^{66} \mathrm{Zn}$

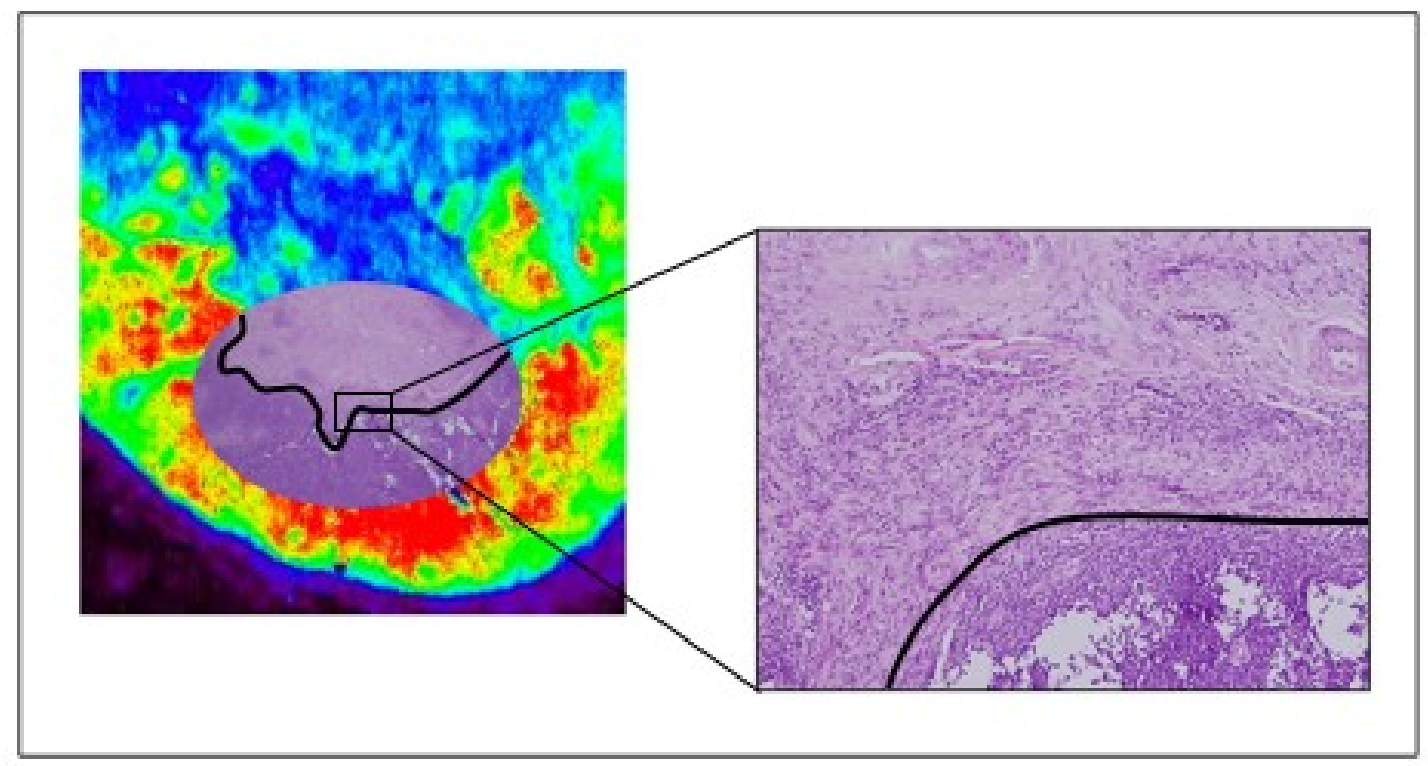

Figure 2: Overlayed 31P image and photomicrograph of lymph node showing boundary of normal and metastatic melanoma cells.

Five further lymph nodes containing metastatic melanoma were imaged in a similar way and are shown in Figures 3 and 4. The trends discussed above were confirmed in each of the samples. The central node in Figure 3(a) displayed only a very small melanoma micrometastasis on the H\&E stained section and elemental images clearly identified the presence of tumour cells. The heightened contrast between metastasis and non-tumourous normal lymphoid tissue in ${ }^{31} \mathrm{P} /{ }^{66} \mathrm{Zn}$ images was confirmed as shown in Figures 3 and 4. 


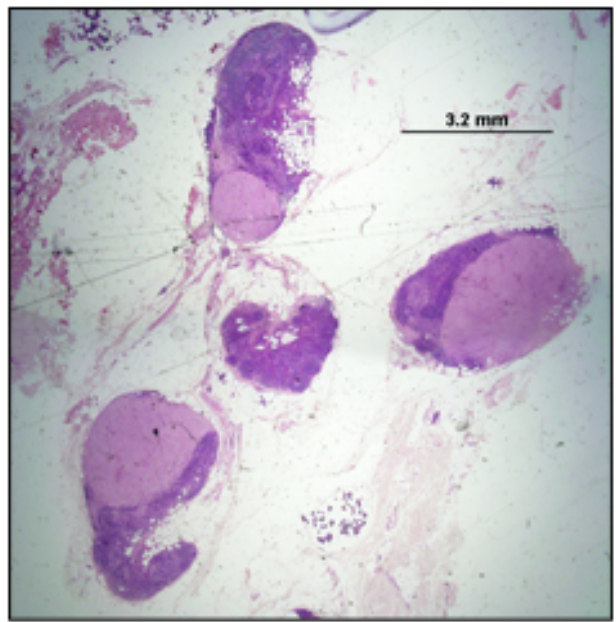

(a) H \& E Stain

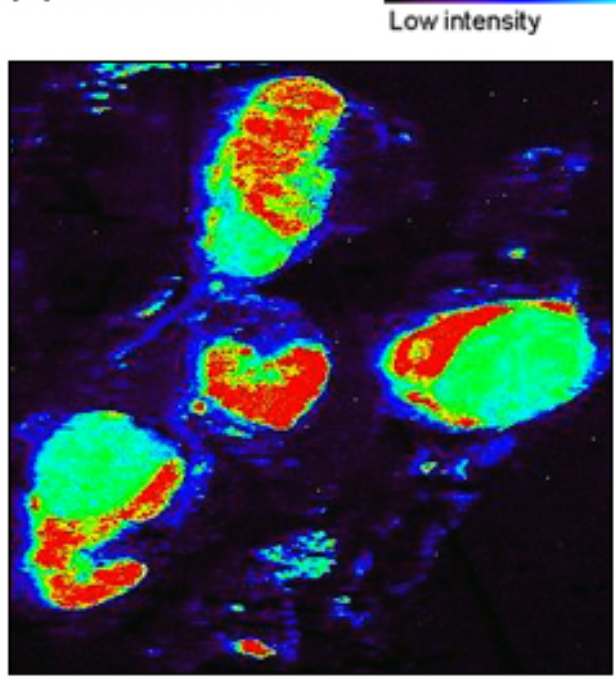

(c) ${ }^{31} \mathrm{P}^{+} / 34 \mathrm{~S}^{+}$

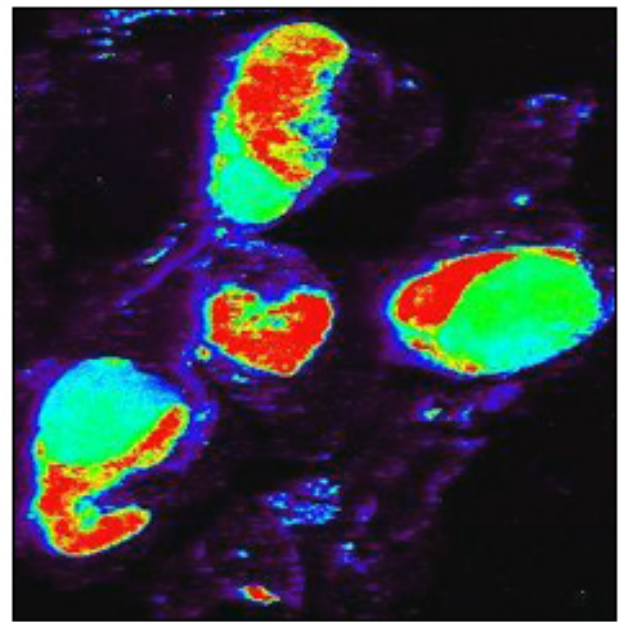

(b) ${ }^{31} \mathrm{P}^{+}$

High intensity

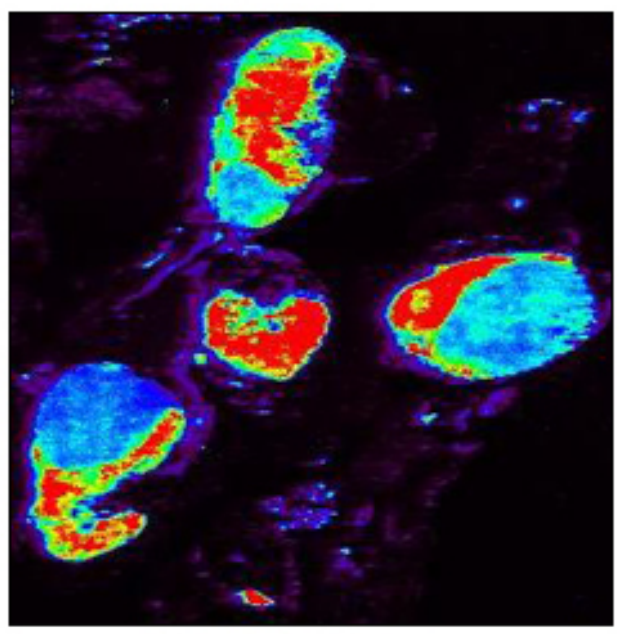

(e) ${ }^{31} \mathrm{P}^{+} / 66 \mathrm{Zn}^{+}$

Figure 3: Five lymph nodes each partly involved by metastatic melanoma. (a) $\mathrm{H}+\mathrm{E}$ stain, (b) ${ }^{31} \mathrm{P}$ image, (c) ${ }^{31} \mathrm{P} /{ }^{34} \mathrm{~S}$ and (d) ${ }^{31} \mathrm{P} /{ }^{66} \mathrm{Zn}$ 

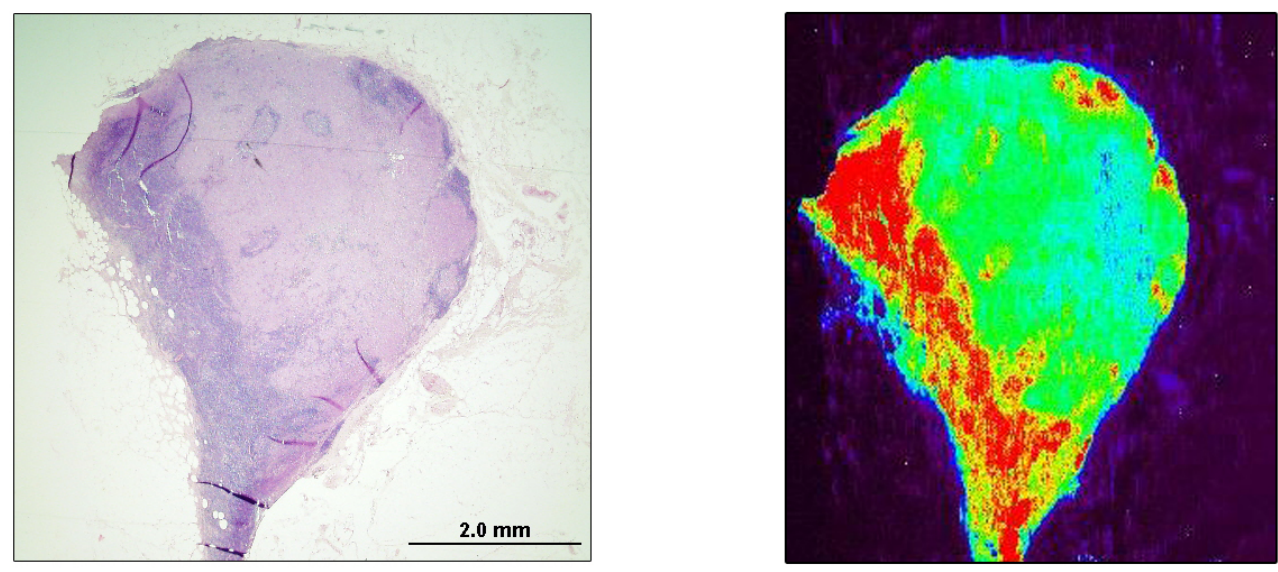

(a) H \& E Stain
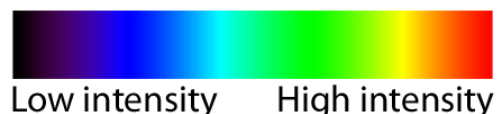

(b) ${ }^{31} \mathrm{p}^{+}$

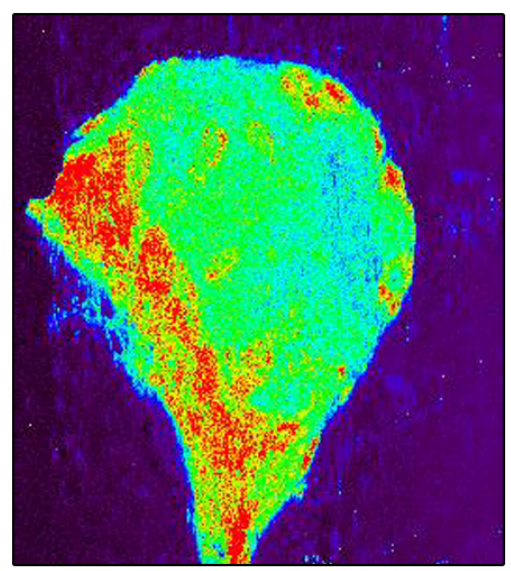

(c) ${ }^{31} \mathrm{p}^{+} / 34 \mathrm{~S}^{+}$

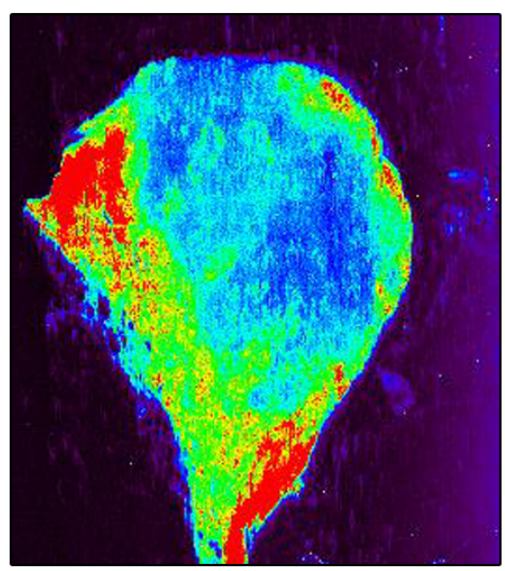

(d) ${ }^{31} \mathrm{p}+/ 66 \mathrm{Zn}+$

Figure 4: Lymph node partly replaced by metastatic melanoma. (a) $\mathrm{H}+\mathrm{E}$ stain (the tumour cells represent the pale area involving the upper right portion of the lymph node), (b) ${ }^{31} \mathrm{P}$ image, (c) ${ }^{31} \mathrm{P} /{ }^{34} \mathrm{~S}$ and (d) ${ }^{31} \mathrm{P} /{ }^{66} \mathrm{Zn}$

\section{Conclusions}

Elemental bio-imaging was used to identify and map metastatic melanoma within single lymph nodes according to relative trace element concentrations. There was an excellent correlation between decreased relative phosphorus concentration and the presence of the tumour, which was confirmed by comparison with standard histopathology. Additionally, images of trace element ratios provided further visual information about malignancy within the nodes. With further refinement it may be possible to apply this method to very small lymph node samples, such as those obtained by fine needle biopsy, to determine whether they contain melanoma metastatses. This could obviate the need for surgical excision biopsy and formal histopathology of lymph nodes and other tissues to determine whether they contain metastases and potentially may have a role in the diagnosis of primary tumours. 


\section{References}

1. World Health Organization, Solar ultraviolet radation: Global burden of disease from solar ultraviolet radiation, WHO, Geneva, 2006.

2. Australian Institute for Health and Welfare, Cancer survival and prevalence in Australia: cancers diagnosed from 1982 to 2004, AIHW, Canberra, 2003.

3. $\quad$ M. B. Lens and M. Dawes, Brit J Dermatol, 2004, 150, 179-185.

4. J. F. Thompson, R. A. Scolyer and R. F. Kefford, Lancet, 2005, 365, 687-701.

5. D. L. Morton, J. F. Thompson and B. B. R. Kroon, Textbook of Melanoma, Martin Dunitz, London, 2004.

6. Z. Hammody, S. Argov, R. K. Sahu, E. Cagnano, R. Moreh and S. Mordechai, Analyst, 2008, 133, 372-378.

7. $\quad$ P. Lasch and D. Naumann, Cell Mol Biol, 1998, 44, 189-202.

8. E. Ly, O. Piot, R. Wolthuis, A. Durlach, P. Bernard and M. Manfait, Analyst, 2008, 133, $197-$ 205.

9. D. R. Bandura, O. I. Ornatsky and L. Liao, J Anal Atom Spectrom, 2004, 19, 96-100.

10. M. G. Cherian, A. Jayasurya and B.-H. Bay, Mutat Res, 2003, 533, 201-209.

11. M. Bergomi, G. Pellacani, M. Vinceti, S. Bassissi, C. Malagoli, D. Alber, S. Sieri, L. Vescovi, S. Seidenari and R. Vivoli, J Trace Elem Med Biol, 2005, 19, 69-73.

12. M. R. Ros-Bullon, P. Sanchez-Pedreno and J. H. Martinez-Liarte, Melanoma Res, 1998, 8, 273-277.

13. R. M. Caprioli, T. B. Farmer and J. Gile, Anal Chem, 1997, 69, 4751-4760.

14. D. S. Cornett, M. L. Reyzer, P. Chaurand and R. M. Caprioli, Nat Methods, 2007, 4, 828-833.

15. J. Feldmann, A. Kindness and P. Ek, J Anal Atom Spectrom, 2002, 17, 813-818.

16. M. V. Zoriy, M. Dehnhardt, G. Reifenberger, K. Zilles and J. S. Becker, Int J Mass Spectrom, 2006, 257, 27-33.

17. R. W. Hutchinson, A. G. Cox, C. W. McLeod, P. S. Marshall, A. Harper, E. L. Dawson and D. R. Howlett, Anal Biochem, 2005, 346, 225-233.

18. M. V. Zoriy, M. Dehnhardt, A. Matusch and J. S. Becker, Spectrochim Acta B, 2008, 63, 375382.

19. M. Zoriy, A. Matusch, T. Spruss and J. S. Becker, Int J Mass Spectrom, 2007, 260, 102-106.

20. R. A. Scolyer, L.-X. L. Li, S. W. McCarthy, H. M. Shaw, J. R. Stretch, R. Sharma and J. F. Thompson, Melanoma Res, 2004, 14, 263-268. 